\title{
Endometrial stromal sarcoma together with leiomyosarcoma in a single patient: a rare case report
}

\author{
Reema Jain*, Rita Ranjan, Poonam Agarwal
}

Department of Obstetrics \& Gynaecology, DDU hospital, Delhi, India

Received: 30 January 2016

Revised: 14 March 2016

Accepted: 15 March 2016

\section{*Correspondence:}

Dr. Reema Jain,

E-mail: reemaforfb@gmail.com

Copyright: () the author(s), publisher and licensee Medip Academy. This is an open-access article distributed under the terms of the Creative Commons Attribution Non-Commercial License, which permits unrestricted non-commercial use, distribution, and reproduction in any medium, provided the original work is properly cited.

\begin{abstract}
Uterine sarcomas are relatively rare tumors of mesodermal origin. The three most common histological variants of uterine sarcoma are Leiomyosarcomas, Endometrial stromal sarcomas, and Carcinosarcomas. It is extremely uncommon to find the 2 variants in a single patient. Given the rarity of these tumors there are limited reports in the literature referring to the clinical management and final outcome of these cases. Our patient was a 60 year old postmenopausal women presented in the opd with post-menopausal bleeding. Ultrasound report showed a fibroid of around 8 by $8 \mathrm{~cm}$ fundal fibroid. Endometrial biopsy report showed low grade endometrial sarcoma while the CECT report showed leiomyosarcoma creating a dilemma in the diagnosis. Patient was then taken up for surgical exploration. The biopsy report confirmed the existence of both the types of tumor, a very rare finding.
\end{abstract}

Keywords: Post-menopausal bleeding, Sarcoma, Ultrasound

\section{INTRODUCTION}

Uterine sarcomas are rare uterine malignancies and constitute 2 to $5 \%$ of all uterine malignancies. ${ }^{1}$ Most of the patients lack symptoms or present with a rapidly enlarging pelvic mass. Out of the three common histological variants endometrial stromal sarcoma (15\% of all uterine sarcomas) occurs commonly in perimenopausal women. The most frequent symptoms are abnormal uterine bleeding, pelvic pain, and pelvic mass. The median age of leiomyosarcoma (40\% of all sarcomas) is somewhat lower than that of other sarcomas. Presenting symptoms which are of short duration include rapidly increasing abdominal lump, vaginal bleeding or pelvic pain. The incidence of sarcomatous changes in benign leiomyoma are reported between $0.13 \%$ to $0.8 \%{ }^{2}$ The third common variant is carcinosarcoma, which is composed of mixture of sarcoma and carcinoma. ${ }^{3}$ Almost all of the carcinosarcomas occur after menopause, at a median age of 62 years. It is considered to be highly malignant as the disease extends outside the uterus in 40 to $60 \%$ of cases at the time of diagnosis.
Staging of uterine sarcomas is carried out using the updated FIGO system for uterine sarcomas, taking into account that uterine sarcomas are different from endometrial cancer. ${ }^{4}$

The first important step in the treatment is surgical exploration. Total abdominal hysterectomy along with bilateral salpingo-oophrectomy is the treatment of choice. Any visible growth should be taken up for biopsy. Lymphadenectomy should be done in endometrial stromal sarcoma and carcinosarcoma, but may be omitted in leiomyosarcoma, in which the risk of lymph node metastasis is low and therapeutic and diagnostic value is questionable.

\section{CASE REPORT}

Our patient was a 60 year old post-menopausal women presented in OPD with the complain of post-menopausal bleeding and lump abdomen. On per speculum examination slight bleeding from OS seen. On per 
vaginal examination her uterus was irregularly enlarged to 18 weeks size firm in consistency and bilateral fornices free. Her ultrasound report showed 8 by $6 \mathrm{~cm}$ fundal fibroid with necrotic foci. Her endometrial biopsy was done and the report came out to be low grade endometrial stromal sarcoma. Her CECT report showed a complex solid cystic mass of around 12 by $10 \mathrm{~cm}$ arising from fundus with possibility of uterine leiomyosarcoma. Patient was admitted in gynae ward; all investigations sent and were prepared for major surgery. Surgical exploration was done with total abdominal hysterectomy with bilateral salpingo-oophorectomy. No lymphadenopathy was found but pelvic and para aortic lymph node sampling was done. No any other site of tumor invasion was seen during surgery. The histopathology report confirmed the existence of low grade stromal sarcoma along with leimyosarcomatous changes in fundal fibroid without lymph node involvement. Post operatively patient was not given any adjuvant therapy considering the benign nature of low grade tumors (Figure 1,2).

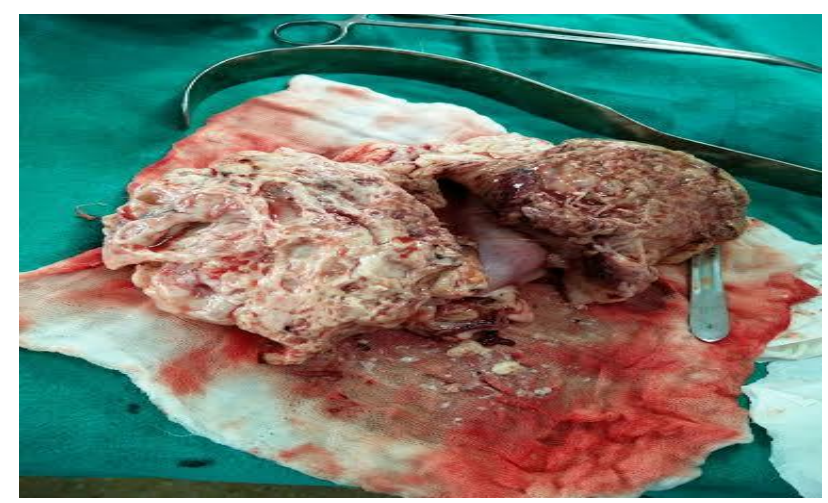

Figure 1: Cut section of the uterus.

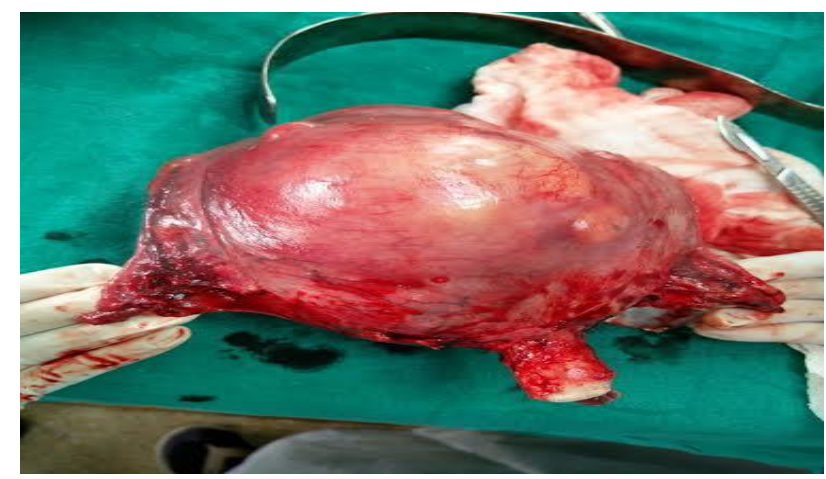

Figure 2: 14 weeks size uterus with multiple seedling fibroid.

\section{DISCUSSION}

Uterine sarcomas are rare and comprise only $3 \%$ of all uterine cancers. Within the group of adult soft tissue sarcomas, they account for $\sim 7 \%$ of new cases. They consist of several distinct histological subtypes.
Uterine Leiomyosarcoma is the most frequent uterine sarcoma and accounts for $\sim 40 \%$ of all uterine sarcomas. ${ }^{5}$ Standard treatment in localized disease is abdominal hysterectomy. Bilateral salpingo-oophorectomy and lymphadenectomy have no proven value in Leiomyosarcomas. The largest published series analysed 3650 patients with uterine sarcomas from the National Oncology Database. In the subgroup of patients with localized Leiomyosarcomas, 131 had received postoperative pelvic irradiation, whereas 398 had not been treated with radiotherapy. The 5-year local recurrence rate was reduced from $16 \%$ to $2 \%$ by radiation without translating into better overall survival. ${ }^{6}$ Conclusion, postoperative pelvic radiation is not generally recommended in leiomyosarcoma. The role of adjuvant chemotherapy remains controversial until results from randomized trials become available.

Endometrial stromal sarcomas (ESS) make up to $\sim 10 \%-$ $15 \%$ of all uterine sarcomas. ${ }^{5}$ Local therapy consists of total abdominal hysterectomy. Given the hormonal responsiveness of most tumors, salpingo-oophorectomy is generally recommended even in the absence of randomized data. They are usually hormone receptor positive, which allows endocrine therapy in most of the cases. Medroxyprogesterone acetate and aromatase inhibitors, in particular, are highly effective and lead to sustained disease control in most cases. ${ }^{7}$ Due to a rather low aggressiveness of the disease and a lack of improvement in overall survival, postoperative radiation is not recommended, although a reduction in local recurrence has been described. ${ }^{6}$

Our patient's biopsy report confirmed the presence of low grade stromal sarcoma and low grade leiomyosarcoma without any evidence of lymph node or other metastasis. No any post-operative adjuvant theory was required hence she was discharged comfortably and was kept for regular follow up in the cancer clinic for early detection of any residual disease.

\section{CONCLUSION}

Uterine sarcomas are uncommon and their differential diagnosis from typical submucosal uterine myomas or benign endometrial polyps could be difficult. The hysteroscopic features of uterine sarcomas are often similar to those of endometrial polyps or submucosal myomas. The histological examination of the specimen is necessary to exclude malignancy and establish the final diagnosis. Total abdominal hysterectomy, bilateral salpingo-oopherectomy with or without pelvic lymphadenectomy is the optimal treatment in such cases. Adjuvant radiation and chemotherapy continues to be evaluated for the treatment.

\section{Funding: No funding sources Conflict of interest: None declared Ethical approval: Not required}




\section{REFERENCES}

1. Brooks SE, Zhan M, Cote T, Baquet CR. Surveillance, epidemiology, and end results nalysis of 2677 cases of uterine sarcoma 1989-1999. Gynecol Oncol. 2004;93(1):204-8.

2. Dinh TA, Oiva EA, Fuller AF, et al. The treatment of Leiomyosarcomas: results from a 10 year experience. Gynecol Oncol. 2004;92(2):648-52.

3. Inthasorn P, Carter J, Valmadre S. Analysis of clinicopathological factors in malignant mixed mullerian tumors of the uterine corpus. Int J Gynecol Cancer. 2002;12(4):348-53.
4. FIGO Committee on Gynecologic Oncology. FIGO staging of uterine sarcoma. Int J Gynecol Obst, 2009.

5. D'angelo E, Prat J. Uterine sarcomas: a review. Gynecol Oncol. 2010;116:131-9.

6. Sampath S, Schultheiss TE, Ryu JK. The role of adjuvant radiation in uterine sarcomas. Int $\mathbf{J}$ Radiat Oncol Biol Phys. 2010;76:728-734.

7. Pink D, Lindner T, Mrozek a, et al. Harm or benefit of hormonal treatment in metastatic low-grade endometrial stromal sarcoma: single centre experience with 10 cases and review of the literature. Gynecol Oncol. 2006;101(3):464-9.

Cite this article as: Jain R, Ranjan R, Agarwal P. Endometrial stromal sarcoma together with leiomyosarcoma in a single patient: a rare case report. Int J Reprod Contracept Obstet Gynecol 2016;5:1239-41. 